\title{
Mathematical model for simulation of defects under a material surface applied to thermographic measurements
}

\author{
by I. Boras, S. Svaic and A. Galovic
}

Faculty of Mechanical Engineering and Naval Architecture, University of Zagreb, 10000 Zagreb, Croatia

\begin{abstract}
We present the mathematical model for simulation the heat conduction process in objects with buried defects. The model is based on the control volume numerical method. The simulation was carried out for defined start and boundary conditions on the model of known geometry and defects (cylindrical holes in different depths). The analysis was carried out in time and amplitude domain. The results of the thermographic measurements taken on the real model having the same characteristic are given too. From the thermograms and numerical simulation the geometry of the defects is determined by means of the inverse procedure.
\end{abstract}

\section{Introduction}

The TNDT method is based on the thermal response of a specimen in space and time domain $\vartheta=\vartheta(x, y, z, t)$. The problem of defect detection under the material surface can be solved experimentally and theoretically. The experiment is based on thermographic measurement of the temperature distribution on the reference surface in time. The theoretical part is related to the solution of the heat conduction problem in $1 D, 2 D$ or $3 D$ co-ordinates in time domain [1].

The defects under the surface are regions where the thermodynamical properties change considerably so that the temperature distribution on the surface is affected. In the theoretical part of this paper the numerical method (the method of control volume) is applied. Theoretical and experimental results are compared.

\section{Geometrical characteristic of the model and parameters of the process}

The real model with defects under the material surface is made of steel with known thermodynamical properties. The defects are in a form of cylinders with equal diameters located in different depths. Fig. 1 shows the ground plate with defects. The analysis is carried out for parameter - current contrast defined with equation (1), [2]:

$$
C_{r}=\frac{T_{n d}(t)-T_{d}(t)}{T_{n d}(t)}
$$

and the results of the analysis are presented in the amplitude and time domain.

\section{Results}

\subsection{The results of the experiment}

Fig. 2 shows the experimental setup. The thermal stimulation of the object is obtained with constant heat flux (using a lamp of $500 \mathrm{~W}$ ). The model is located in the frame with isolation to avoid heat loses from the side of the model. The surface temperature distributions are registered by the thermography camera "AGA 680 STANDARD" in defined 
time steps. On the model there are two thermocouples in different depths. They monitor the temperatures at reference positions and allow for a comparison with the numerical results.

The experimental results of the surface temperature distribution in defined time steps are presented on series of thermograms (Fig. 3 at $240 \mathrm{~s}$ ). The time when the current contrast reaches its maximum is the key parameter for the analysis because it is the measure for the defect depth. This conclusion corresponds to published results [3].

\subsection{The results of numerical simulation}

The numerical analysis is based on the equation of heat conduction in Cartesian coordinates (2):

$$
\rho c \frac{\partial \vartheta}{\partial t}=\frac{\partial}{\partial x}\left(\lambda \frac{\partial \vartheta}{\partial x}\right)+\frac{\partial}{\partial y}\left(\lambda \frac{\partial \vartheta}{\partial y}\right)+\frac{\partial}{\partial z}\left(\lambda \frac{\partial \vartheta}{\partial z}\right)+\phi_{v}
$$

By implicit discretisation of equation (2) it is obtained [4]:

$$
a_{P} \vartheta_{p}=a_{E} \vartheta_{E}+a_{W} \vartheta_{W}+a_{N} \vartheta_{N}+a_{S} \vartheta_{S}+a_{T} \vartheta_{T}+a_{B} \vartheta_{B}+b
$$

For each control volume the set of algebraic equations must be solved taking into consideration the start and boundary conditions (to be the same as in the experiment).

The results of the numerical simulation are the surface temperature distributions in defined time steps and corresponding contrast curves. Fig. 4 shows the surface temperature distribution at $240 \mathrm{~s}$.

The analysis of the results leads to the following conclusions [5]:

- the time of maximum contrast is related to the defect depth,

- the time of maximum contrast is influenced by the presence of other defects,

- in the case of more defects the time of maximum contrast depends on the surface point chosen for contrast calculation,

- in the case of only one defect the dependence between the time of maximum contrast and the chosen position disappears.

The relation between the time of maximum contrast and the defect depth is shown in fig. 5 .

\subsection{Comparison between experimental and numerical results}

The comparison is done between the temperatures measured by thermocouples and those calculated numerically. The correspondence between the results can be seen in fig. 6 .

\section{Inverse procedure}

The preliminary analysis of the temperature distribution on the specimen surface by amplitude allows to locate the defect under the specimen surface and to determine the size. The unknown dimension of the defect is the dimension perpendicularly to the observed surface. This dimension can be evaluated using the inverse procedure by the analysis of the surface temperature distribution around the defect or by the temperatures on defined positions near the defect. In this case the numerical simulation starts with supposition of the unknown dimension and must be corrected in each step. The correction is based on the difference between real and calculated temperature for the same position. It is necessary to repeat this procedure since the differences between measured and calculated temperatures reach the demanded accuracy. The principle of the inverse procedure is shown in fig. 7 .

\section{Conclusion}

The numerical model of the heat conduction in the specimen containing the under surface defects can be a useful tool for the analysis of all influencing parameters (heat flux, 
dimensions of the defects, material properties, etc) on the results as well as their consequences on the specimen surface temperature distribution. Combination of thermography and numerical simulation gives the new possibilities in development of the TNDT methods.

\section{REFERENCES}

[1] ALMOND (D. P.) and PATEL (P. M.) - Photothermal Science and Technique, London, Chapman \& Hall, 1996.

[2] VAVILOV (V.) - Transient thermal NDT: conception in formulae, Quantitative Infrared Thermography, QUIRT 92, Chatenay-Malabry, France, 1992, p. $229-234$.

[3] FAVRO (L. D.), HAN (X.), KUO (P. K.) and THOMAS (R. L.) - Imaging the early time behaviour of reflected thermal-wave pulses, Thermosense XVII, An International Conference on Thermal Sensing and Imaging Diagnostic Applications, Orlando, Florida, USA, 1995, p. 162 -166.

[4] PATANKAR (S. V.) - Numerical Heat Transfer and Fluid Flow, Hemisphere Publishing Corporation, Washington, 1980.

[5] BORAS (I.) and SVAIC (S.) - Determination of the Defect Parameters in Specimen by Means of Thermography and Numerical Methods, Proceeding of The International Society for Optical Engineering, San Antonio, Texas, USA, 1998, Vol. 3396, p. 271-281.

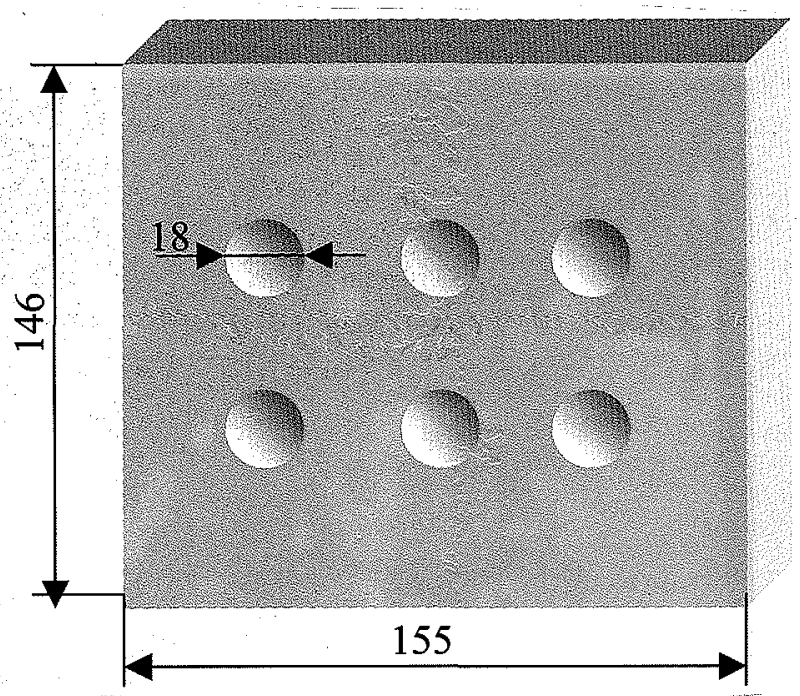

Fig. 1. The ground plate with defects 
http://dx.doi.org/10.21611/qirt.1998.006

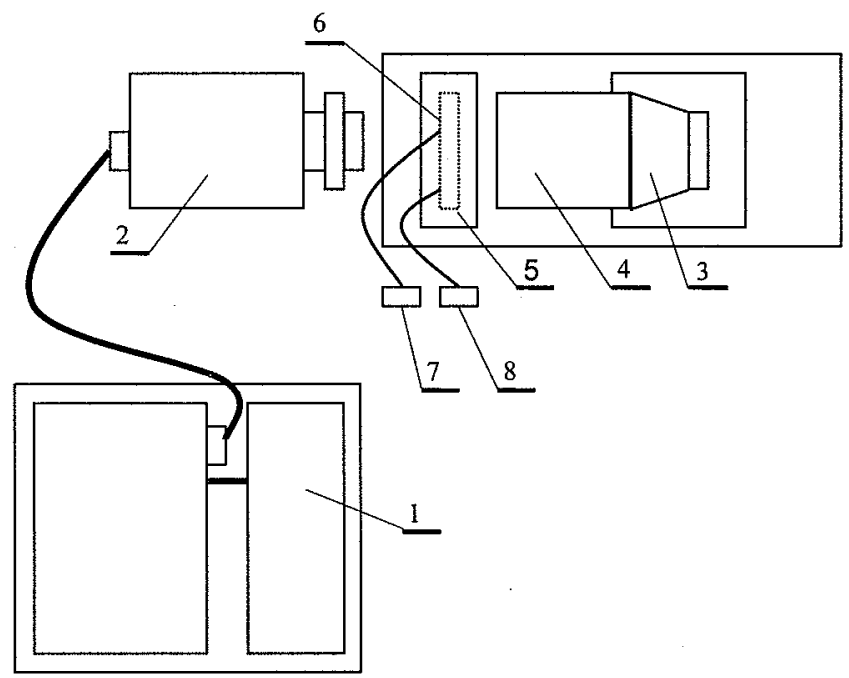

Fig. 2. The experimental rig; 1 - thermographic equipment, 2 - infrared camera, 3 lamp, 4 - canal, 5 - isolation, 6 - model, 7 - thermocouple on depth $12 \mathrm{~mm}, 8$ termocouple on surface

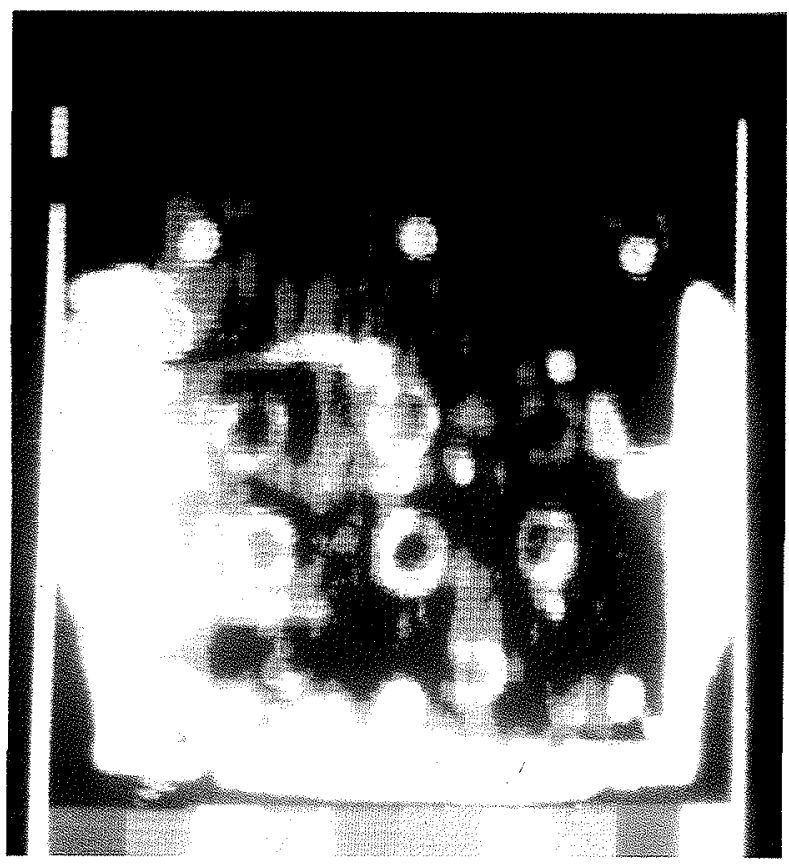

Fig. 3. Thermogram at $240 \mathrm{~s}$ 
http://dx.doi.org/10.21611/qirt.1998.006

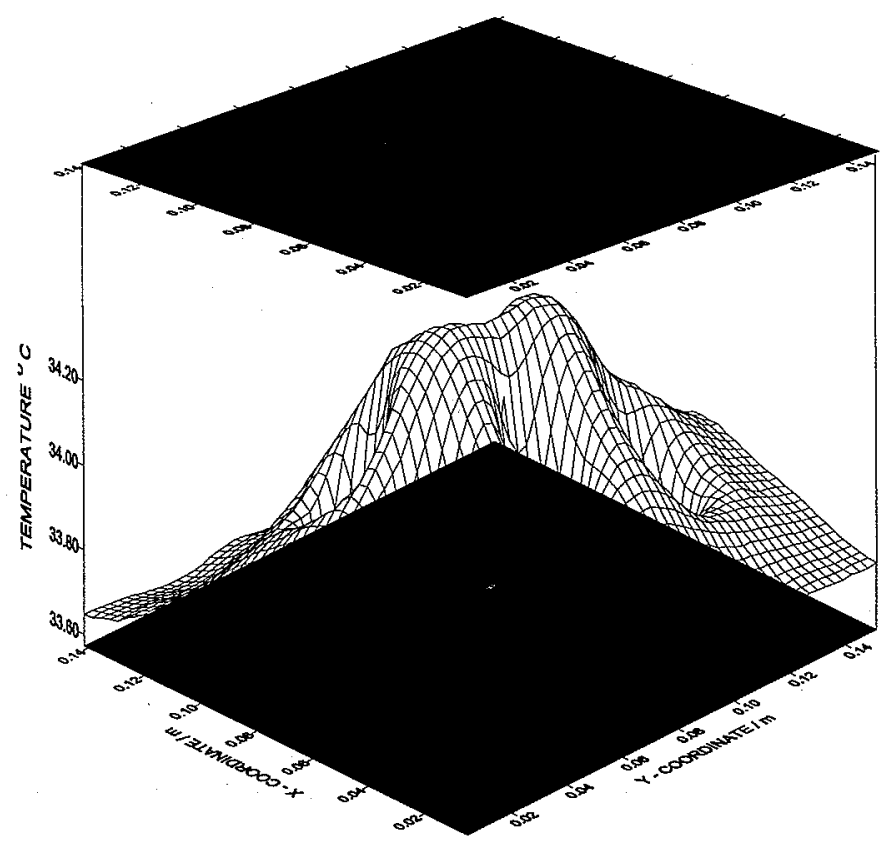

Fig. 4. Surface temperature distribution at $240 \mathrm{~s}$

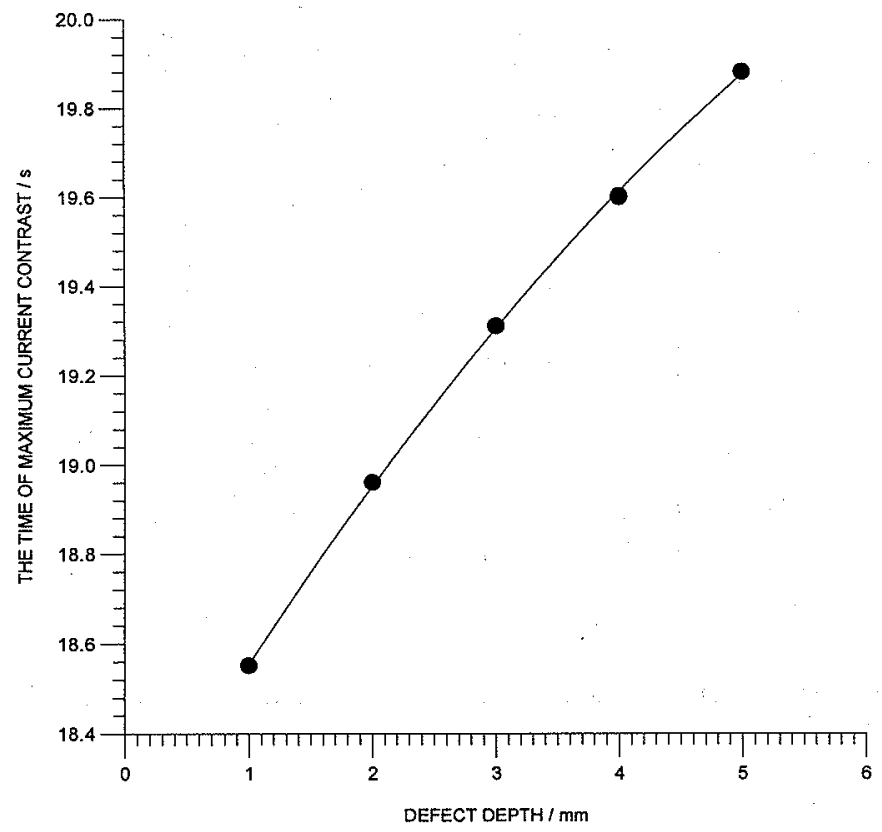

Fig. 5. The time of maximum current contrast versus defect depth 
http://dx.doi.org/10.21611/qirt.1998.006

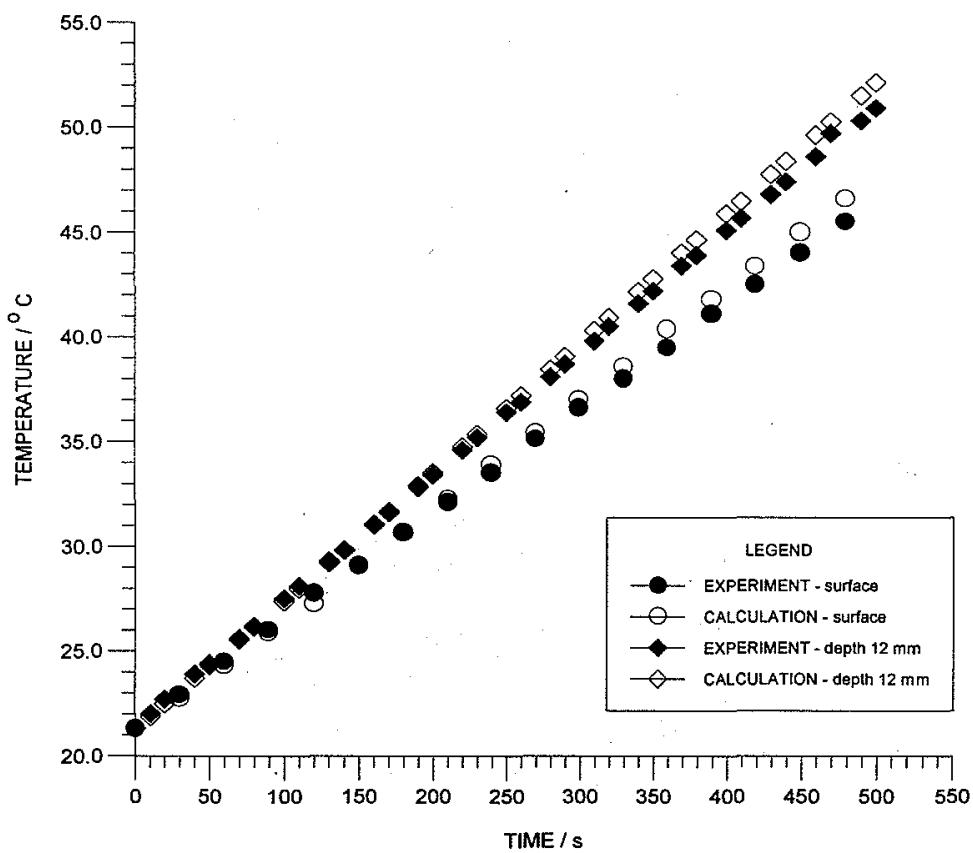

Fig. 6. Comparison between measured and calculated temperatures on defined positions

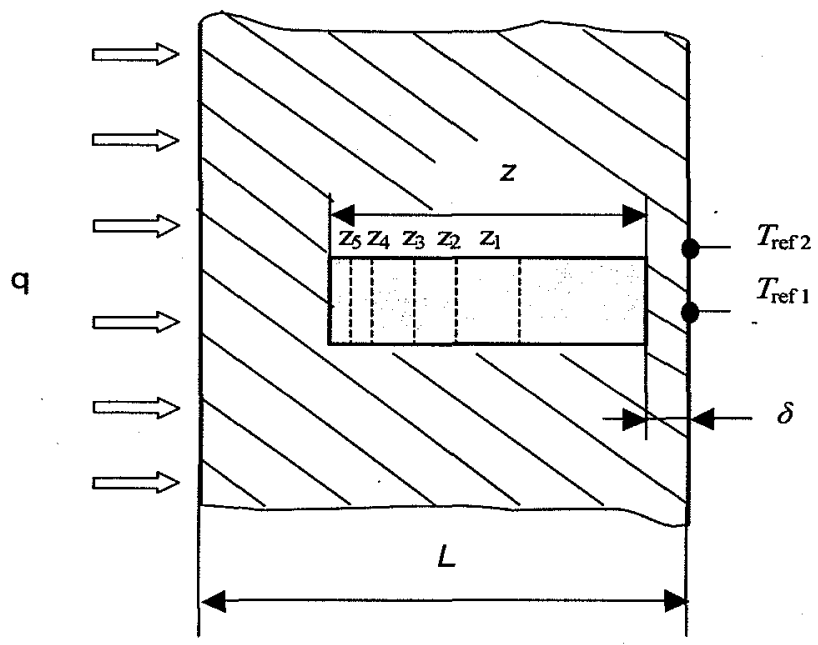

Fig. 7. Determination of the defect dimension $\mathbf{z}$ by inverse procedure 